\title{
Cáncer de laringe inicial cirugía transoral con láser
}

\section{Initial stages of laryngeal cancer. Laser transoral surgery}

\author{
Dr. Luis Zanolli de $\mathrm{S}^{*}$, Dr. Rodrigo Pinto $\mathrm{C}^{\star}$, Dra. Maritza Rahal E*.
}

\begin{abstract}
RESUMEN
日 cáncer de laringe constituye una de las patologías malignas más frecuentes de la vía aérea digestiva superior, contando hoy en día con la cirugía clásica, la radioterapia y la cirugía transoral con láser como tratamiento curativo. $\theta$ uso de la cirugía láser transoral exclusiva para el tratamiento curativo de los estadios iniciales, constituye una alternativa segura y eficaz en el control local, recidiva y preservación laríngea de la enfermedad con resultados comparables a la radioterapia.
\end{abstract}

Palabras clave: Cáncer Laringe, Láser.

\section{SUMMARY}

One of the most frequent malignant pathologies of the upper respiratory and digestive tracts is laryngeal cancer. Nowadays, it's treated using classical surgery, radiotherapy and transoral laser surgery. The treatment of the initial stages of larynx cancer by transoral laser surgery has provided a safe and efficient alternative for recidivation and local disease control and laryngeal preservation, with results that are comparable to those of radiotherapy.

Key words: Laryngeal cancer, laser.

\section{INTRODUCCIÓN}

Se realizó una revisión y análisis del uso de cirugía transoral con láser en cuanto a su indicación, control local, sobrevida específica como objetivos primarios en el cáncer de laringe inicial generando una búsqueda de las bases de datos electrónicas Medline desde 1966 hasta el 2000, Embase desde 1980 hasta 2000, Ginal desde 1982 hasta octubre del 2000 y Cancerlit desde 1963 hasta 2000 y en el registro Cochrane de Ensayos Controlados.

日 cáncer de laringe corresponde a uno de los tumores malignos más frecuentes de la vía aérea digestiva superior (VADS), calculándose su incidencia en aproximadamente 9.000 nuevos casos en $\mathbb{E} U U$ en el 2002 y 120.000 casos nuevos en el mundo cada año; destacando una mayor incidencia en hombres, teniendo al tabaco y al alcohol uno de

\footnotetext{
* Servicio Aorrinolaringología, Hospital Barros Luco Trudeau.
} 
los principales factores de riesgo ${ }^{1-2}$. En la gran mayoría de los casos corresponde al tipo epidermoide (más del 95\%), preferentemente en la glotis y su presentación en estadios iniciales de la enfermedad es cerca de 3 de cada 4 casos $^{3}$; donde la radioterapia, la cirugía clásica o endoscópica con o sin láser son métodos validados con similares tasas de control local, conservación laríngea y sobrevida específica de enfermedad a 5 años ${ }^{3}$.

日 uso del láser en la cirugía laríngea se origina en EUUU. a fines de los sesenta, durante los siguientes años se produce el desarrollo paralelo de otras tecnologías que facilitan su uso como nuevos materiales endoscópicos y el desarrollo del microscopio acoplado al láser. Son los trabajos de Strong, Jako y Vaunghan a fines de los setenta que inician su uso en lesiones malignas de laringe $e^{4,5}$ (Tabla 1).

\section{INDICACIÓN DE CIRUGÍA ENDOSCÓPICA CON LÁSER}

Uno de los elementos más importantes de definir en el uso de la terapia endoscópica con láser es la posibilidad de una adecuada exposición del campo quirúrgico, esto estará determinado por múltiples factores tales como: los propios del paciente en cuanto a movilidad cervical, tamaño de cavidad oral y sus estructuras como lengua, dientes y mandíbula. Una adecuada posición seráfundamental para la visualización de la comisura anterior ${ }^{6}$. Se incluyen como requisitos para esta técnica contar con los materiales adecuados como son laringoscopios, ópticas, láser, microscopio, tubos endotraqueal resistentes al láser y material quirúrgico antirreflejo.

Hoy en día la cirugía transoral con láser es una alternativa de tratamiento definitivo para el cáncer laríngeo inicial en una sola intervención. Así esos enfermos evitan la radioterapia y sus efectos se cundarios como también un menor costo. Múltiples son los estudios que muestran que los resultados de la técnica endoscópica con láser en cuanto a control local, calidad de la voz, conservación del órgano y sobrevida específica son comparables con los tratamientos convencionales ${ }^{4,7-11}$.

En el uso del láser para la resección transoral se han descrito 4 procedimientos estandarizados ${ }^{12}$ :

1. La disección por debajo del epitelio de la membrana basal en el espacio superficial de la lámina propia superficial.

2. Disección dentro del espacio profundo de la lámina propia superficial.

3. Disección entre la lámina propia profunda de la cuerda vocal y el músculo vocal.

Tabla 1.

\begin{tabular}{|llll|}
\hline Estudio & N. de Pacientes & $\begin{array}{l}\text { Control local } \\
\text { a 5 años (\%) }\end{array}$ & $\begin{array}{l}\text { Conservación } \\
\text { laríngea a 5 años (\%) }\end{array}$ \\
\hline Moreau & 26 Tis, 62 T1a, 24 T1b & $100 \%$ & $100 \%$ \\
Eckel & 161 (casos) & $86,3 \%$ & $91,7 \%$ \\
Peretti & 13 Tis, 75 T1 & $92 \%, 90 \%$ & $94,4 \%,-$ \\
Gallo & 12 Tis, 117 T1a, 22 T1b & $100 \%, 94 \%, 91 \%$ & $100 \%,-,-$ \\
Steiner & 333 T1a & $89,3 \%$ & $97,6 \%$ \\
Pradhan & 52 T1a, 17 T1b, 13 T2 & $90 \%, 65 \%, 77 \%$ & $94 \%, 88 \%, 92 \%$ \\
\hline
\end{tabular}

Esta tabla corresponde a trabajos publicados desde el 2000 en adelante que muestra que la resección transoral con láser es completamente comparable con otros tratamientos en cuanto a resultados de control local y sobrevida en $\mathrm{T}$ in situ y $\mathrm{T} 1^{5-6}$. Pues con radioterapia exclusiva para los $\mathrm{T} 1$ y $\mathrm{T} 2$ se logra un control local de $70 \mathrm{a}$ $85 \%$, una sobrevida de hasta $85 \%$ y una conservación de la laringe de hasta $87 \%{ }^{3}$. 
4. Disección de la musculatura tiroaritenoídea ${ }^{13}$.

La Sociedad Europea de Laringología ha realizado una estandarización de las cordectomía presentando la clasificación de las cordectomías endoscópicas que se presentan en la Figura 1.

\section{RESULTADOS EN LASER V/S RADIOTERAPIA EN T2 (Tabla 2)}

La sexta edición del TNM clasifica a los tumores T2 en una amplia gama de características en relación a extensión local y pronóstico, es por esto que la decisión endoscópica debe ser tomada caso a caso analizando tanto las variables del paciente como las propias del tumor. Esta situación es considerada fundamental por los distintos autores para asegurar un adecuado resultado oncológico; de esta manera, Peretti et al ${ }^{6}$ concluye que los T2 que dan un rango de control local de entre $79 \%-100 \%$ considera aquellos que tienen extensión superficial a supra o subglotis (porción lateral) o con compromiso del compartimiento del músculo vocal pero sin invasión de la grasa medial a la lámina tiroidea (Tabla 3).

日 compromiso de la comisura anterior es un tema de gran debate, resultando en todo un desafío para el otorrino. Su sólo compromiso contraindicó el uso del láser para algunos autores a comienzo

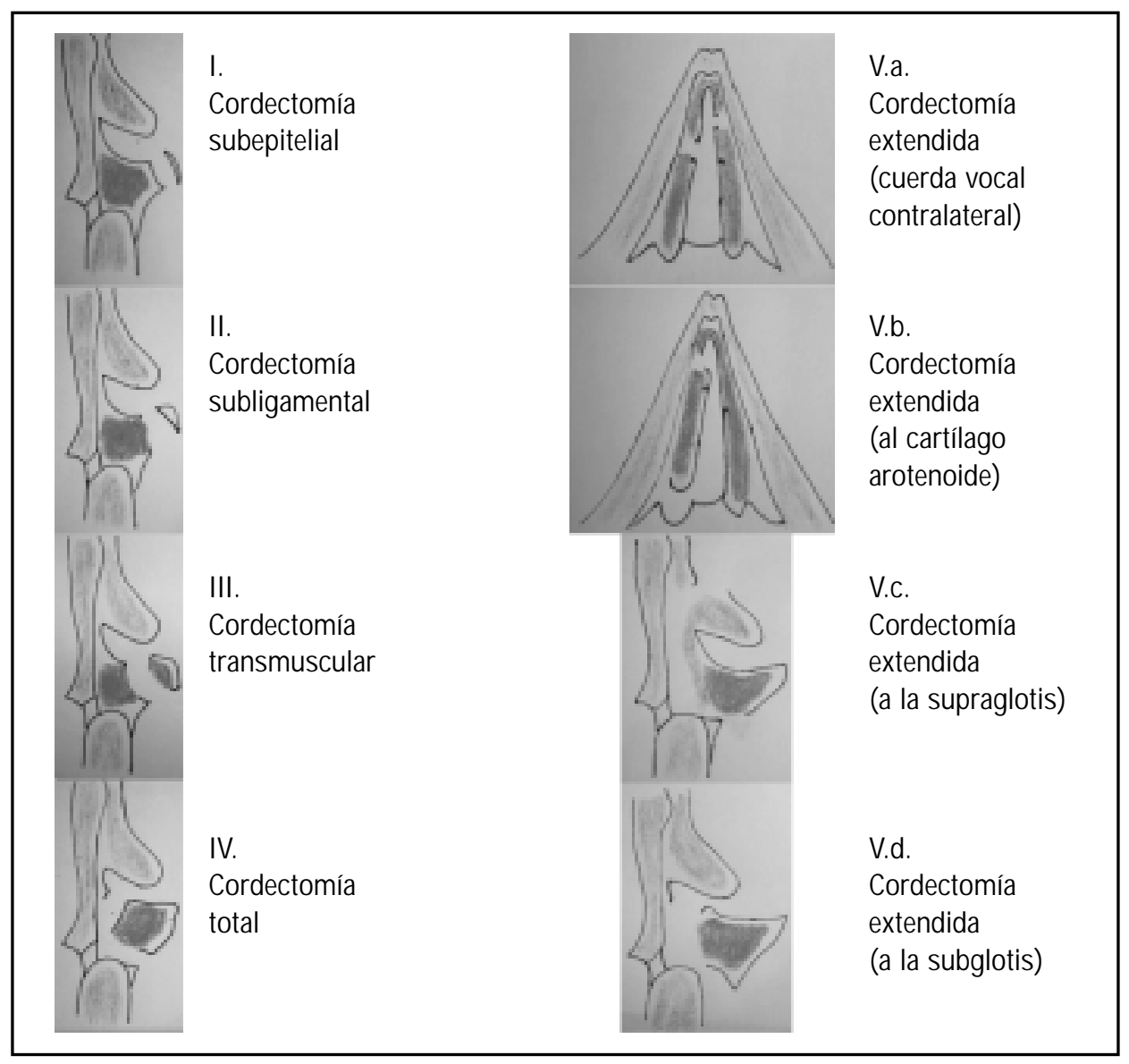

Figura 1. Resultados en Láser v/s Radioterapia en T1. 
Tabla 2.

\begin{tabular}{|lccc|}
\hline Cáncer de Laringe T1 & Control local a 5 años & Sobrevida & Sobrevida específica \\
\hline Girugía Láser Endoscópica & $83-93 \%$ & $85-99 \%$ & $96-99 \%$ \\
Radioterapia & $85-94 \%$ & $70-88 \%$ & $93-98 \%$ \\
\hline
\end{tabular}

Se muestra el resultado en porcentajes de 20 estudios en cuanto a control local a 5 años, sobrevida y sobrevida específica a 5 años en los $\mathrm{T} 1$ de laringe en tratamiento con láser vía endoscópica versus radioterapia ${ }^{6}$. De aquí se desprende que el láser tendría indicación en T1.

Tabla 3.

\begin{tabular}{|lccc|}
\hline Cáncer de Laringe T2 & Control local a 5 años & Sobrevida & Sobrevida espećfica \\
\hline Cirugía Láser Endoscópica & $73-89 \%$ & $68-83 \%$ & $83-97 \%$ \\
Radioterapia & $68-80 \%$ & $64-78 \%$ & $70-88 \%$ \\
\hline
\end{tabular}

Se muestra el promedio de porcentajes de 20 estudios en cuanto a control local a 5 años, sobrevida y sobrevida específica a 5 años en los T2 de laringe en tratamiento con láser vía endoscópica versus radioterapia ${ }^{5}$.

de los noventa ${ }^{14,15}$. Avances recientes han permitido una mejor exposición para una resección completa. La laringectomía parcial vertical endoscópica permite la exposición de la comisura anterior o el acceso al espacio paraglótico posterolateralmente al remover la cuerda vocal falsa. En cuanto a los resultados no hay consenso pues existen trabajos como el de Pradhan y cols ${ }^{16}$ que reportó una recurrencia de $27,7 \%$ para pacientes resecados con láser y compromiso de comisura anterior versus $13 \%$ para pacientes con el mismo estadío que no lo tenían. Este reporte se contrasta con el de Zeitels y cols ${ }^{18}$ que muestra que el compromiso de comisura anterior no afecta en una mayor recurrencia Aun considerando las diferencias de estos autores, la extensión del compromiso de la comisura anterior a supra o subglotis en el control local con láser exclusivamente cae a $68 \%$, lo que es comparable a los valores obtenidos con la radioterapia exclusiva ${ }^{18-23}$ o a una laringectomía frontolateral abierta ${ }^{24-26}$ pero significativamente peor a la obtenida con la laringectomía supracricoídea especialmente con quimioterapia neoadyu- vante $^{27-30}$. En países donde el uso del láser es de uso rutinario y la primera elección presentan resultados optimistas a pesar del compromiso de la comisura anterior. En España Bernal reporta una serie de 54 enfermos con compromiso de comisura anterior seguidos por una media de 40 meses con sólo 4 enfermos $(7,5 \%)$ con recidiva de los cuales 3 terminaron en laringectomía total.

Se debe considerar que los carcinomas de la comisura anterior tienen un margen muy pequeño para ser etapificados desde T1 cuando son superficiales hasta T4 al invadir el cartílago y del mismo modo no es lo mismo un carcinoma de cuerda vocal que invada la cuerda contralateral a través de la comisura anterior, que normalmente es un cáncer superficial a que sea propiamente de la comisura anterior, que normalmente es más invasivo.

Aro elemento a considerar es la subetapificación en los T2. Una vez operado el enfermo en forma endoscópica y analizada la muestra en anatomía patológica, si existe invasión del espacio paraglótico se re-etapifica en T3 determinando resultados no tan optimistas ${ }^{6}$. 
Desde una mirada técnica el uso de la cirugía láser trae consigo la dificultad de los cambios histológicos producidos en los tejidos, de esta manera el patólogo se ve enfrentado a un artefacto en las piezas a analizar sobretodo lo que hace relación con el margen resectivo y margen tumoral, este artefacto se ve aumentado si se realiza congelación de la muestra para estudio contemporáneo por lo cual este no se recomien$\mathrm{da}^{31}$. Manteniendo el principio de que la obtención de márgenes quirúrgicos libres de enfermedad es el requisito prioritario para el éxito de la cirugía oncológica.

La discusión en la indicación de láser en T2 es aun controversial; los buenos resultados sólo se rán obtenidos con una buena elección del paciente. Es por esto que no es el TNM el que posibilita o no la indicación de la cirugía transoral con láser sino que la adecuada exposición y el compromiso o no de elementos vitales.

\section{Láser en supraglotis y subglotis}

En el manejo de los tumores de supraglotis a fines de los setenta Vangha ${ }^{6}$ reintrodujo el manejo endoscópico. Se acepta que en general las lesiones que involucren la epiglotis suprahioidea, pliegues aritenoepiglóticos y cuerda vocal falsa son ideales para la resección con láser así como lesiones en la epiglotis infrahioidea. La extensión subglótica del tumor por más de $1 \mathrm{~cm}$ por debajo de la comisura anterior sería una contraindicación por la imposibilidad de una adecuada exposición tangencial ${ }^{6}$. Desde los promisorios resultados de Steiner en $1993^{32}$ en adelante se han publicado numerosos estudios donde se obtienen resultados alentadores como el publicado por Motta y $\operatorname{cols}^{33}$ de 106 pacientes de T1, T2 y T3 (todos N0) con una sobrevida de $91 \%, 88 \%$ y $81 \%$ respectivamente y preservación laríngea superior al $85 \%$. Hoy en día se acepta que los resultados obtenidos con la cirugía láser en tumores supraglóticos son comparables a los que se consiguen con métodos resectivos clásicos, sobre todo a lo que hace referencia al control local y a la sobrevida.

\section{Conservación de la voz, complicaciones $y$ costos en el tratamiento con láser}

Sabemos que la comunicación es un factor importante a la hora de determinar calidad de vida, con respecto a esto, existen distintas opiniones sobre la superioridad de la radioterapia comparado con la cirugía endoscópica láser. Esto es claro cuando hablamos de laringectomía con resección importante; pero no lo es tanto cuando lo comparamos con una cirugía minimamente invasiva. En un estudio de Juhani Pukander, sólo $2,5 \%$ de los pacientes no pudo usar su voz normalmente, en cambio $70 \%$ quedó satisfecho con su calidad de voz. Otro estudio, que también comparó calidad de voz en cirugía láser mínimamente invasiva, específicamente en el tratamiento de carcinoma glótico T1a, mostró que no existieron diferencias en calidad de voz en comparación a la radioterapia $^{34}$. Ésta misma conclusión se puede obtener en otros reportes publicados en los últimos años ${ }^{35-40}$.

Las complicaciones posoperatorias, también pueden ser comparadas en relación a las complicaciones posradioterapia; en este aspecto existen reportes de algunas complicaciones posquirúrgicas como enfise ma subcutáneo, hematoma subcután $\Theta^{40}$, daño térmico, necrosis, etc ${ }^{41-43}$; los que en todo caso no pueden ser comparables con la morbilidad asociadaa la radio necrosis. Bajo estas condiciones, la radiotera pia podría ser innecesaria cuando se tiene la alternativa de la cirugía minimamente invasiva ${ }^{44}$.

$日$ aspecto financiero también ha sido considerado en la literatura mundial; el aspecto más destacable es el tiempo de hospitalización y de necesidad de licencia médica, algunos estudios muestran un tiempo de hospitalización en un rango de 2 a 6 días, con un promedio de 2,3 días ${ }^{40}$, pudiendo el paciente volver a una actividad normal en 1-2 semanas; por lo que los cálculos indican que la radioterapia full dosis seria el doble de cara $^{46}$. Esta situación no sólo lo haría más conve niente para el paciente, sino que también al sistema público de salud.

En nuestro medio existen ya publicaciones inicia les del uso de láser en tumores laringeos mostrando favorables resultados funcionales y oncológicos ${ }^{47}$. 


\section{DISCUSIÓN}

Hoy en día, cada vez existe más evidencia publicada de la utilización efectiva del láser por vía endoscópica como el tratamiento único del cáncer de laringe en estadio precoz; si bien no contamos con trabajos prospectivos randomizados que determinen una evidencia "tipo uno" 47 , existen múltiples reportes y artículos originales que analizan comparando el control local, sobrevida, conservación laríngea, calidad de voz y costos que nos permiten afirmar que en los estadios iniciales como es el tumor in situ y el T1 los resultados obtenidos son comparables con las otras técnicas aceptadas. Sin embargo no se ha descrito un aumento de las tasas de complicaciones posoperatorias en la cirugía parcial de rescate en los casos de falla del tratamiento endoscópico con láser a diferencia de lo que ocurre luego de la falla de la radioterapia ${ }^{48}$.

Fundamental resulta, que los estudios de etapificación previos a la cirugía deben seguir protocolos de análisis de imágenes y ópticos con esfuerzos a evitar la sub-etapificación lo que determinaría una mala elección de la técnica y posterior resultados desfavorables condición especialmente orientada a los cánceres etapificados como T2 cuando esta misma técnica quirúrgica se aplica en T3 (reclasificados en el posoperatorio), los resultados finales no son alentadores.

De este modo, del análisis se desprende que la técnica endoscópica con láser es una técnica cada vez más desarrollada por diversos autores, mostrando resultados comparables con las demás técnicas convencionales para los cánceres iniciales de laringe siempre y cuando se realiza una adecuada selección del enfermo que radica más en la adecuada exposición del tumor más que en la clasificación del TNM, no existiendo, en estos estadios compromiso de estructuras que contraindique su realización.

\section{BIBLIOGRAFÍA}

1. LE DJ. Definitive radiotherapy for squamous carcinoma of the larynx. Aolaryngol Cin North Am 2002; 32: 1013-33.
2. The Royal College of Surgeons of England, London. Effective Head \& Neck Cancer Management: Second Consensus Document 2000, British Association of Otorhinolaryngologists, Head and Neck Surgeons.

3. BACK G, SoOD $S$. The management of early laryngeal cancer: options for patients and therapists. Ourr Opin Otolaryngol Head Neck Surg 2005; 13(2): 85-91. Review.

4. STRONG MS, JAKO GJ. Laser surgery in the larynx: early clinical experience with continuous 002 laser. Ann Otol Rhinol Laryngol 1972; 81: 791-8.

5. VAUGHAN CW, STRONG MS, JAKO GJ. Laryngeal carcinoma: transoral treatment utilizing the CO2 laser. Am J Surg 1978; 136 (4): 490-3.

6. Peretr G Piazza C, Bozoni A. Endoscopic treatment for early glottic cancer: indications and oncologic outcome. Otolaryngol Cin North Am 2006; 39 (1): 173-89. Review.

7. BLAKESLEI D, VAUGHN CW, SHAPSHAY SM, SIMPSON GT, STRONG MS. Excisional biopsy in the selective management of $\mathrm{T} 1$ glottic cancer: a three-year follow-up study. Laryngoscope 1984; 94: 488-94.

8. SHAPSHAY SM, HYBES RL, BOHGIAN RK. Laser excision of early vocal cord carcinoma: indications, limitations, and precautions. Ann Otol Rhinol Laryngol 1990; 99: 46-50.

9. CRAGLES, BRANDENBURGJ. Laser cordectomy or radiotherapy: cure rates, communication, and cost. Otolaryngol Head Neck Surg 1993; 108 : 648-54.

10. RYDel $R$, SCHalen L, FEX S, EUNER A. Voice evaluation before and after laser excision vs. radiotherapy of T1A glottic carcinoma Acta Oolaryngol 1995; 115: 560-5.

11. Peretti G, Piazza C, Balzanelli C, Cantare LLA G, NICOLAI P. Vocal outcome after endoscopic cordectomies for Tis and T1 glottic carcinomas. Ann Otol Rhinol Laryngol 2003; 112: 174-9.

12. YeAGR LB, GRILLONE GA. Organ preservation surgery for intermediate size (T2 and T3) laryngeal cancer. Otolaryngol Cin North Am 2005; 38(1): 11-20, vii. Review. 
13. Z曰تte-S SM. Phonomicrosurgical treatment of early glottic cancer and carcinoma in situ. Am J Surg 1996; 172(6): 704-9.

14. KRESPI YP, MenTZR CJ. Laser surgery for vocal cord carcinoma involving the anterior commissure. Ann Otol Rhinol Laryngol 1989; 98(2): 105-9.

15. WOLANSBrRger M, DORT JC. Endoscopic laser surgery for early glottic carcinoma: a clinical and experimental study. Laryngoscope 1990; 100(10 Pt 1): 1100-5.

16. PRADHAN SA, PAI PS, N⿴囗十 SI, D'CRUZ AK. Transoral laser surgery for early glottic cancers. Arch Aolaryngol Head Neck Surg 2003; 129(6): 623-5.

17. Zete-S SM, HILLMAN RE, FRANCO RA, BUNTING GW. Voice and treatment outcome from phonosurgical management of early glottic cancer. Ann Otol Rhinol Laryngol Suppl 2002; 190: 3-20.

18. Mendenhall WM, Amdur RJ,MorRis CG et al. T1-T2NO squamous cell carcinoma of the glottic larynx treated with radiation therapy. $J$ Cin Oncol 2001; 19: 4029-36.

19. Jergensen K, Godballe C, Hansen O et al. Cancer of the larynx: treatment results after primary radiotherapy with salvage surgery in a series of 1005 patients. Acta Oncol 2002; 41: 69-76.

20. Johansen lV, Grau C, Overgaard J. Gottic carcinoma: patterns of failure and salvage treatment after curative radiotherapy in 861 consecutive patients. Radiother Oncol 2002; 63: 257-67.

21. JoHANSEn LV, Grau C, OVergaARD J. Laryngeal carcinoma: multivariate analysis of prognostic factors in 1252 consecutive patients treated with primary radiotherapy. Acta Oncol 2003; 42: 771-8.

22. Garden AS, Forster K, Wong PFet Al. Results of radiotherapy for T2NO glottic carcinoma: does the "2" stand for twice-daily treatment? Int J Radiat Oncol Biol Phys 2003; 55: 322-28.

23. COLASANTO JM, HAFTIY BG, WILSON LD. Evaluation of local recurrence and second malignancy in patients with $\mathrm{T} 1$ and $\mathrm{T} 2$ squamous cell carcinoma of the larynx. Cancer J 2004; 10: 61-6.

24. Lacoourreye O, Wensten G, Brasnu D et al. Vertical partial laryngectomy: a critical analysis of local recurrence. Ann Otol Rhinol Laryngol 1991; 30: 357-62.

25. LACOOURREYEO, GIT́́RREZ-FONSECA R, GaRCíA D ET AL. Local recurrence after vertical partial laryngectomy, a conservative modality of treatment for patients with stage I-II squamous cell carcinoma of the glottis. Cancer 1999; 85: 2549-56.

26. LaCOOURREYE O, LaCOOURREYE L, GarCiA D et AL. Vertical partial laryngectomy versus supracricoid partial laryngectomy for selected carcinomas of the true vocal cord classified as T2NO. Ann Otol Rhinol Laryngol 2000; 109: 965-71.

27. LACOOURREYE O, MUSCATELO L, LACCOURREYE L ET AL. Supracricoid partial laryngectomy with cricohyoidoepiglottopexy for "early" glottic carcinoma classified as T1-T2NO invading the anterior commisure. Am J Colaryngol 1997; 18: 385-90.

28. Chevalier D, Lacoourreye O, Brasnu D et al. Oricohyoidoepiglottopexy for glottic carcinoma with fixation or impaired motion of the true vocal cord: 5-year oncologic results with 112 patients. Ann Otol Rhinol Laryngol 1997; 106: 364-9.

29. LacoOURREye O, Diaz EM JR, Bassot V et al. A multimodal strategy for the treatment of patients with $\mathrm{T} 2$ invasive squamous cell carcinoma of the glottis. Cancer 1999; 85: 40-6.

30. LaCoOURREYE O, Bassot V, Brasnu D Et al. Chemotherapy combined with conservation surgery in the treatment of early larynx cancer. Ourr Opin Oncol 1999; 11: 200-3.

31. BERMAL M, BLANOH JL, VILASECA I, STEN⿴囗十 W, AMBROsCH P. Grugía con Láser 002 en la vía

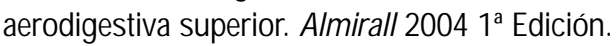

32. STENER W. Results of curative laser microsurgery of laryngeal carcinomas. Am J Otolaryngol 1993; 14: 116-21.

33. MOTTA G, ESPOSITO E, TESTA D, IOVINE R, MOTtA S. COR laser treatment of supraglottic cancer. Head Neck 2004; 26 (5): 442-6. 
34. Petiris J, VAN GOCh C, GOOR K, Verdonckb I et AL. Health status and voice outcome after treatment for T1a glottic carcinoma. Eur Arch Otorhinolaryngol 2004; 261 (10): 534.

35. Tamura E Kitahara S, OGura M et al. Voice Quality After Laser Surgery or Radiotherapy for T1a Gottic Carcinoma. Laryngoscope 2003; 113 (5): 910-4.

36. Kelmann A, Bergler W, Artzt M, Hogmann K. Vocal function following laser and conventional surgery of small malignant vocal fold tumours. Journal of Laryngology and Otology 1996; 110 (12): 1138-41.

37. MOGUIRT WF, BLALOCK D, KOUFMAN JA ET AL. Comparative voice results after laser resection or irradiation of $\mathrm{T} 1$ vocal cord carcinoma. Archives of Aolaryngology-Head and Neck Surgery 1994; 120: 951-5.

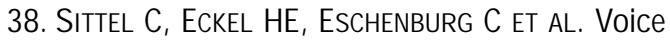
quality following endolaryngeal laser surgery. Laryngology, Rhinology and Otology 1998; 77: 219-25.

39. HiranoM, HIRADE Y, KaWASAKI H. Vocal function following carbon dioxide laser surgery for glottic carcinoma Annals of Aology, Rhinology and Laryngology 1985; 94 (3): 232-5.

40. SIGSTON E, DE MONES E, BABIN E Early-Stage Gottic Cancer Oncological Results and Margins in Laser Cordectomy. Arch Otolaryngol Head Neck Surg 2006; 132(2): 147-52.

41. Zeteles SM, Franco RA, Dailey SH, et al. Office-based treatment of glottal dysplasia and papillomatosis with the 585-nm pulsed dye laser and local anesthesia. Ann Otol Rhinol Laryngol 2004; 113(4): 265-76.

43. Franco RA, ZetEles SM, FarinelLI WA, Et AL. 585-NM pulsed dye laser treatment of glottal papillomatosis. Ann Otol Rhinol Laryngol 2002; 111: 486-92.

44. Franco RA, Zeteles SM, Farinell WA et aL. 585-nm pulsed dye laser treatment of glottal dysplasia Ann Otol Rhinol Laryngol 2003; 112(9 Pt 1): 751-8.

45. Gallo A, M. Vincentis M, Manciocoo V et al. CO2 laser cordectomy for early-stage glottic cancer: A long-term follow-up of 156 cases. Laryngoscope 2002; 112: 370-4.

46. CRAGLES, BRANDENBURGJ. Laser cordectomy or radio-therapy: Oure rates, communication, and cost. Otolaryngol Head Neck Surg 1993; 108: 648-54.

47. ORTEGA AT, Olcese LT, ZANOLI LD, LILLO SD. Manejo endoscópico de la patología maligna inicial. Rev Otorrinolaringol Oir Cabeza Cuello 2005; 65: 197-202.

48. Dey P, Arnold D, Wight R, MacKenZIE K, Kelly C, WILSON J. Radiotherapy versus open surgery versus endolaryngeal surgery (with or without laser) for early laryngeal squamous cell cancer. Cochrane Database Syst Rev 2002; (2): CD002027. Review.

49. JOHANSEN LV, GRAU C, OVERGARD J. Laryngeal carcinoma - multivariate analysis of prognostic factors in 1252 consecutive patients treated with primary radiotherapy. Acta Oncol 2003; 42(7): 771-8. 